\title{
Design of Sealable Custom-Shaped Cell Mimicries Based on Self- Assembled Monolayers on CYTOP Polymer
}

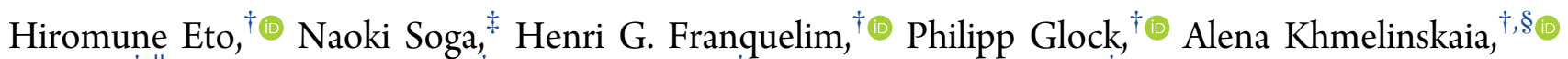 \\ Lei Kai, ${ }^{\dagger, \|}$ Michael Heymann, ${ }^{\dagger}$ Hiroyuki Noji, ${ }^{\ddagger}$ and Petra Schwille* ${ }^{* \dagger}{ }^{\dagger}$ \\ ${ }^{\dagger}$ Max Planck Institute of Biochemistry, Am Klopferspitz 18, D-82152 Martinsried, Germany \\ ${ }^{\ddagger}$ Department of Applied Chemistry, Graduate School of Engineering, The University of Tokyo, Bunkyo-ku, Tokyo 113-8656, Japan \\ ${ }^{\S}$ Institute for Protein Design, University of Washington, Seattle 98195, Washington, United States \\ "School of Life Sciences, Jiangsu Normal University, Shanghai Road 101, 221116 Xuzhou, P. R. China
}

\section{Supporting Information}

\begin{abstract}
In bottom-up synthetic biology, one of the major methodological challenges is to provide reaction spaces that mimic biological systems with regard to topology and surface functionality. Of particular interest are cell- or organelleshaped membrane compartments, as many protein functions unfold at lipid interfaces. However, shaping artificial cell systems using materials with nonintrusive physicochemical properties, while maintaining flexible lipid interfaces relevant to the reconstituted protein systems, is not straightforward. Herein, we develop micropatterned chambers from CYTOP, a less commonly used polymer

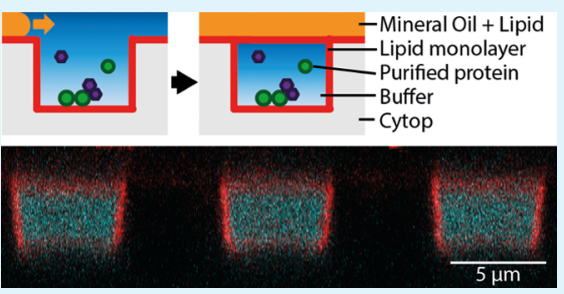
with good chemical resistance and a refractive index matching that of water. By forming a self-assembled lipid monolayer on the polymer surface, we dramatically increased the biocompatibility of CYTOP-fabricated systems. The phospholipid interface provides an excellent passivation layer to prevent protein adhesion to the hydrophobic surface, and we succeeded in cell-free protein synthesis inside the chambers. Importantly, the chambers could be sealed after loading by a lipid monolayer, providing a novel platform to study encapsulated systems. We successfully reconstituted pole-to-pole oscillations of the Escherichia coli MinDE system, which responds dramatically to compartment geometry. Furthermore, we present a simplified fabrication of our artificial cell compartments via replica molding, making it a readily accessible technique for standard cleanroom facilities.
\end{abstract}

KEYWORDS: micropatterning, bottom-up biology, lab-on-a-chip, synthetic biology, self-assembled monolayers, lipids, polymer, CYTOP

\section{INTRODUCTION}

Functional encapsulation of biochemical reactions inside artificial biomimetic compartments is one of the most fundamental challenges in bottom-up synthetic biology. Many cellular reactions depend dramatically on volume size and topology as well as surface properties of the reaction spaces they are enclosed to. Often, binding to and from membranes, membrane insertion, or membrane transformation is part of their cellular functionality. Mimicking membraneenclosed compartments of a particular shape and at the same time retaining structural flexibility are a daunting task that has so far not been conclusively solved. On the one hand, there is a strong motivation to maintain the classical advantages of in vitro techniques, such as high throughput, controllability, and accessibility of experiments. Examples of preferred compartments include water-in-oil droplets and liposomes, which can be produced in large numbers and feature cell-sized volumes with phospholipid mono- or bilayer interfaces. Through their mimicry of the cellular phospholipid interface, these compartments can support not only cytosolic reactions, but also those that involve membranes. ${ }^{1,2}$ On the other hand, deforming them to any other shape than their equilibrium spherical geometry remains a challenge, ${ }^{3,4}$ and thus, there is an ongoing quest to develop new platforms that support the custom design of reaction spaces other than spheres. ${ }^{5,6}$ In this regard, the most prominent developments have been through microfabrication and photolithography techniques to pattern materials into any custom shape, including cell mimicries. ${ }^{6-8}$ However, their effectiveness in biological studies depends greatly on the biocompatibility of the materials used to fabricate such structures. Cytotoxicity, poor compatibility with reagents, and a large refractive index mismatch with water (rendering their analysis by common microscopy techniques problematic) are notorious problems faced with commonly used materials. At the same time, standardized production routines and reasonable costs should support the development into a robust, widely accessible platform. In the past years, one of the most widely used polymers for the design of biomimetic reaction spaces has been PDMS (polydimethylsiloxane). Prepolymer of PDMS is commercially available, inexpensive, and easy to prepare. It is also biocompatible and unreactive. Membrane-coated PDMS chambers have been successfully

Received: March 21, 2019

Accepted: May 28, 2019

Published: May 28, 2019 
employed to mimic the elongated shape of bacteria for the reconstitution of oscillatory dynamics, resulting from the selforganization of geometry-sensitive MinCDE proteins involved in bacterial cell division. ${ }^{10}$ However, constructing fully membrane-enclosed reaction volumes based on PDMS support ${ }^{7}$ is tedious and bears the risk of artifact generation under non-ideal optical conditions.

In this study, we turn our attention to CYTOP, an amorphous perfluorinated polymer, whose unique physicochemical properties make it a material for a wide range of applications. In particular, with its low autofluorescence and refractive index $(n=1.34)^{11}$ similar to that of water $(n=1.33)$, CYTOP can be readily used in combination with optical microscopy. ${ }^{12}$ It has high chemical resistance and therefore a high compatibility with many reagents, including organic solvents and oils that could otherwise dissolve or swell conventionally used microfabrication materials, such as PDMS. $^{13}$ As a hydrophobic material, it has a notably low surface energy $\left(19 \mathrm{mN} \mathrm{m}^{-1}\right),{ }^{11}$ which makes it a useful coating material as a release layer in microfluidic applications. ${ }^{14,15}$

Compartmentalized structures fabricated with CYTOP have been used for biological applications in the past. Particularly noteworthy are the attoliter-sized arrayed lipid bilayer chamber systems (ALBiC), ${ }^{16}$ developed by Noji et al., that are powerful tools to study functions of water-soluble enzymes or membrane-associated proteins. Although CYTOP has an excellent compatibility with many water-soluble proteins, its very low surface energy (and high hydrophobicity) result in enhanced adsorption and, consequently, often denaturation of more hydrophobic proteins coming into contact with the surface. Peripheral membrane proteins, transmembrane proteins, or proteins that generally contain hydrophobic moieties that are either exposed or not well shielded cannot usually be studied in combination with CYTOP surfaces.

To overcome this problem, we developed a supported lipidCYTOP interface, where the CYTOP surface is coated with a phospholipid monolayer. The stable layer of lipids not only provides an effective passivation of the surface against the adsorption of hydrophobic molecules and residues, but also converts the interface into a model membrane platform to study lipid-lipid interactions, the functional role of membrane proteins, membrane-protein interactions, as well as other biochemical processes, such as molecular transport, signaling, and catalysis. ${ }^{17}$

Furthermore, we introduce replica molding to simplify compartment fabrication. ${ }^{18}$ Instead of lithographic patterning of each CYTOP chip independently, our process requires an SU8 master to be prepared in the cleanroom only once, as subsequent replica chips can be patterned using inexpensive tools outside of the cleanroom.

\section{MATERIALS AND METHODS}

Chamber Fabrication by Photolithography. Chambers were patterned as described previously. ${ }^{19}$ CYTOP 816AP (Asahi-Glass, Japan) was coated on a glass cover slide $(32 \mathrm{~mm} \times 24 \mathrm{~mm}$; Matsunami) by spin-coating at $4000 \mathrm{rpm}$ for $30 \mathrm{~s}$ and baked for $1 \mathrm{~h}$ at $180{ }^{\circ} \mathrm{C}$. Note that the different grades of CYTOP used in the subsequent methods differ only in their functional groups, which is important in how they couple to their various substrates (e.g., glass), but not in the overall material properties, such as refractive index, surface energy, or cross-linking density. ${ }^{11}$ The thickness of the CYTOP layer was $3 \mu \mathrm{m}$, as determined by an optical profiler (VK$\mathrm{X} 1000$; Keyence). Photolithography was then performed using a positive photoresist (AZP4903; AZ Electronic Materials) to pattern mask structures on the CYTOP layer. Subsequently, the resistpatterned substrate was dry etched with $\mathrm{O}_{2}$ plasma using a reactive ion-etching system (RIE-10NR; Samco). For photoresist lift-off, the substrate was sonicated in acetone for $10 \mathrm{~min}$ and rinsed with 2propanol and deionized water.

Chamber Fabrication by Replica Molding. Replica-molded CYTOP chamber fabrication was adopted from an X-ray chip fabrication method that used solvent-diluted epoxy resin. ${ }^{18}$ First, an SU8 master was fabricated by standard photolithography methods: on a 2" silicon wafer, a $3 \mu \mathrm{m}$ thickness SU8 3005 (Microchem, USA) was spin coated, subsequently exposed to UV, and developed. To fabricate the stamp, PDMS was mixed with a cross linker in 10:1 weight ratio, cast on the SU8 master, and cured at $75{ }^{\circ} \mathrm{C}$ over $2 \mathrm{~h}$. The PDMS was then peeled off the master. Subsequently, CYTOP 109AE was dropcast on a clean glass cover slide, and the stamp was pressed down with a weight of $\sim 65 \mathrm{mg} \mathrm{mm}^{-2}$. CYTOP was gently cured on a hotplate at $50{ }^{\circ} \mathrm{C}$ over $20 \mathrm{~min}$, until full solvent evaporation. Finally, the stamp was gently peeled off.

Giant Unilamellar Vesicles Preparation and Hemifusion with CYTOP Surface. Giant unilamellar vesicles (GUVs) composed of DOPC (1,2-dioleoyl-sn-glycero-3-phosphocholine), containing additional $0.005 \mathrm{~mol} \%$ Atto655-DOPE (1,2-dioleoyl-sn-glycero-3phosphoethanolamine), were produced by electroformation in polytetrafluoroethylene chambers with platinum electrodes $4 \mathrm{~nm}$ apart, as described previously. ${ }^{20,21}$ Briefly, $6 \mu \mathrm{L}$ of the lipid mixture $(2$ $\mathrm{mg} \mathrm{mL} \mathrm{m}^{-1}$ in chloroform) was spread onto two platinum wires and dried in a desiccator for $30 \mathrm{~min}$. The chamber was then filled with 370 $\mu \mathrm{L}$ of a $250 \mathrm{mOsm} \mathrm{kg}{ }^{-1}$ aqueous solution of sucrose. An AC electric field of $2 \mathrm{~V}$ (RMS) was applied at a frequency of $10 \mathrm{~Hz}$ for $1.5 \mathrm{~h}$, followed by $2 \mathrm{~Hz}$ for $45 \mathrm{~min}$. The obtained solution was diluted 1:10 in volume in $250 \mathrm{mOsm} \mathrm{kg}{ }^{-1}$ sucrose and further diluted 1:7 in volume in buffer $\mathrm{M}(25 \mathrm{mM}$ Tris- $\mathrm{HCl}, 150 \mathrm{mM} \mathrm{KCl}, 5 \mathrm{mM} \mathrm{MgCl}$, $\mathrm{pH} 7.5)$.

A flat CYTOP was prepared by spin-coating a film of CYTOP $(809 \mathrm{M})$; diluted 1:10 in volume in solvent CT-SOLV180, at 3000 rpm for $40 \mathrm{~s}$; and a reaction chamber assembled, as described previously. $^{22}$

Monolayer Preparation on CYTOP Surface. All lipids were purchased from Avanti Polar Lipids (Alabaster, AL, USA), unless otherwise stated. Small unilamellar vesicles (SUVs) composed of DOPC or DOPC/DOPG (1,2-dioleoyl-sn-glycero-3-phospho-(1'-racglycerol)) (7:3 molar ratio), containing additional $0.005 \mathrm{~mol} \%$ Atto655-DOPE (Atto-TEC GmbH, Siegen, Germany), were prepared at a concentration of $4 \mathrm{mg} \mathrm{mL}{ }^{-1}$ in buffer $\mathrm{M}$, as described elsewhere. $^{22}$ Shortly, lipids dissolved in chloroform were dried under a nitrogen stream, and vials were placed in a desiccator to remove residual chloroform for at least $30 \mathrm{~min}$. Afterward, lipids were slowly rehydrated in buffer $\mathrm{M}$ and SUVs were generated by sonication in a water bath (model 1510; Branson) until the solution appeared clear.

To deposit self-assembled monolayers (SAM) on a planar surface, we first spin-coated a film of CYTOP (809M); diluted 1:10 in volume in solvent CT-SOLV180, at $3000 \mathrm{rpm}$ for $40 \mathrm{~s}$; and assembled a reaction chamber, as described previously. ${ }^{22}$ SUVs were added to the chamber at a concentration of $0.5 \mathrm{mg} \mathrm{mL}^{-1}$ in buffer M. After $10 \mathrm{~min}$ incubation at $37^{\circ} \mathrm{C}$ on a heating block, the SAM was gently washed with a total of $600 \mu \mathrm{L}$ buffer S $(25 \mathrm{mM}$ Tris- $\mathrm{HCl}, 150 \mathrm{mM} \mathrm{KCl}, \mathrm{pH}$ $7.5)$ to remove excess vesicles.

CYTOP Chamber Loading and Sealing. A flow cell was constructed, as described elsewhere. ${ }^{19}$ To prepare SAM on the fabricated chamber surface, SUVs were added to the flow cell at a concentration of $0.5 \mathrm{mg} \mathrm{mL}^{-1}$ in buffer M. After $10 \mathrm{~min}$ incubation on a $37^{\circ} \mathrm{C}$ warm heating block, the SAM was washed by exchanging the volume inside the flow cell 3 times with buffer $S$ to remove excess vesicles. After coating the surface with lipids, the desired reaction mixture was flushed in to fill up the chambers.

To seal the chambers, we first prepared a lipid-mineral oil mixture. A DOPC/DOPG mixture (7:3 molar ratio), dissolved in chloroform, was partially evaporated in a glass vial until the meniscus touched the bottom. Mineral oil (Art-Nr. HP50.1; Carl Roth) was added and 

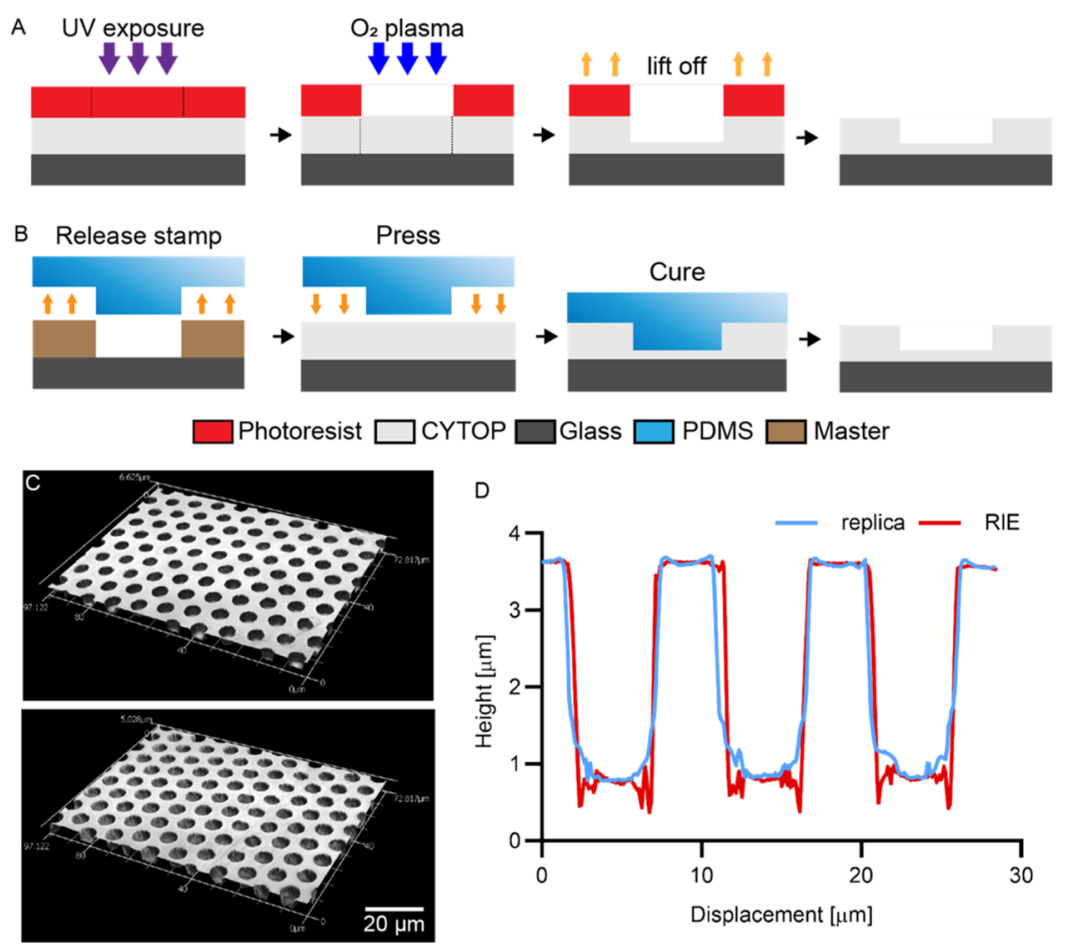

Figure 1. CYTOP chambers microfabricated by (A) photolithography involving reactive ion etching (RIE) and (B) replica molding. Fabricated chambers were imaged on a laser profiler (C, above): RIE; (C, below): replica molded. Chambers were fabricated with height $\sim 3 \mu \mathrm{m}$ and width $\sim 5$ $\mu \mathrm{m}$, and their height profiles are plotted in (D), which reveals that replica-molded chambers can be fabricated with remarkable similarity to those made by RIE.

vortexed, with the final concentration of lipid in the mixture at $2.5 \mathrm{mg}$ $\mathrm{mL}^{-1}$. Handling of mineral oil was done in a nitrogen chamber $(<10 \%$ relative humidity). This mixture was then flushed in to the flow cell to seal the chambers.

Fluorescence Microscopy Imaging and Fluorescence Recovery after Photobleaching. $Z$-stack images of loaded chambers were obtained on a Zeiss LSM780 confocal laser scanning microscope using a Zeiss C-Apochromat $40 \times / 1.20$ water-immersion objective (Carl Zeiss AG, Oberkochen, Germany). Alexa 488 was excited using the $488 \mathrm{~nm}$ argon laser, and Atto655-DOPE using the $633 \mathrm{~nm} \mathrm{He}-$ $\mathrm{Ne}$ laser.

Surface images were taken on Nikon Eclipse Ti2 in a TIRF (total internal reflection fluorescence) set-up using a Nikon Apo TIRF 60×/ 1.4 oil immersion objective, and for imaging chambers, we used Nikon Eclipse Ti in a spinning disk confocal set-up, with the Nikon Plan Fluor 20×/0.75 water immersion objective (both Nikon GmbH, Düsseldorf, Germany). We used the 490 and $640 \mathrm{~nm}$ diode laser lines to image GFP and Atto655-DOPE, respectively. All images were processed using Fiji with the standard set of packaged plugins sufficient for basic analysis. ${ }^{23}$

For fluorescence recovery after photobleaching (FRAP) analysis, a circular spot of $5 \mu \mathrm{m}$ diameter was illuminated with full laser power for $20 \mathrm{~ms}$, and the average intensity was recorded every $3 \mathrm{~s}$ over $300 \mathrm{~s}$. Measurements were taken at room temperature $\left(23^{\circ} \mathrm{C}\right)$. Intensity in a second area of the same dimensions was recorded in the same field of view, in order to correct for the intensity drift. The intensity trace was fit to the equation derived by Soumpasis ${ }^{24}$

$$
f(t)=\mathrm{e}^{-2 \tau_{\mathrm{D}} / t}\left(I_{0}\left(\frac{2 \tau_{\mathrm{D}}}{t}\right)+I_{1}\left(\frac{2 \tau_{\mathrm{D}}}{t}\right)\right)
$$

Here, $\tau_{\mathrm{D}}$ is the characteristic timescale for diffusion, and $t$ is the time. $f(t)$ is the normalized fluorescence, and $I_{0}$ and $I_{1}$ are modified Bessel functions. The diffusion timescale for a bleached spot of radius $r$ is $\tau_{\mathrm{D}}$ $=r^{2} / 4 D$ with $D$ the diffusion coefficient. A custom code written in Python was used for curve fitting and data analysis.
Cell-Free Extract. S30 cell extracts were prepared from either Escherichia coli strain BL21 (DE3) or A19 according to previous protocols described by Kigawa et al. ${ }^{25}$ and Kai et al. ${ }^{26,27}$ The basic cell-free reactions for batch configuration was set up according to previous protocols by Kigawa with modifications as follows: $17 \mathrm{mM}$ of HEPES/KOH (pH 7.5); $3.2 \mathrm{mM}$ of ATP; $1.6 \mathrm{mM}$ of CTP, GTP, and UTP; $1 \mathrm{mM}$ of DTT; $0.175 \mathrm{mg} \mathrm{mL}^{-1}$ of E. coli total tRNA mixture; $0.64 \mathrm{mM}$ of cAMP; $210 \mathrm{mM}$ of potassium glutamate; $8.7 \mathrm{mM}$ of ammonium acetate; $21 \mathrm{mM}$ of magnesium acetate; $34 \mu \mathrm{g} \mathrm{mL}^{-1}$ of L-5formyl-5,6,7,8-tetrahydrofolic acid (folinic acid); $2.0 \mathrm{mM}$ each of 20 amino acids; $2 \%$ PEG (8000); $100 \mathrm{mM}$ of creatine phosphate; 250 $\mathrm{mM}$ of creatine kinase; $15 \mathrm{ng} \mu \mathrm{L}^{-1}$ of plasmid DNA template; $30 \%$ (v/v) of S30 extract from E. coli BL21 (DE3); and $10 \mu \mathrm{g} \mathrm{mL}^{-1} \mathrm{~T} 7$ RNA polymerase.

To estimate the concentration of purified eGFP, an absorption spectrum of a 1:100 dilution in buffer $\mathrm{M}$ was recorded from 300 to $600 \mathrm{~nm}$ using a Jasco V-650 spectrophotometer (Jasco Deutschland $\mathrm{GmbH}$, Germany). The absorption peak at $489 \mathrm{~nm}$ was used in combination with the Lambert-Beer relation, $A=\varepsilon c l$ ( $A=$ measured absorption, $\varepsilon=55000 \mathrm{M}^{-1} \mathrm{~cm}^{-1}$ for eGFP, path length $=10 \mathrm{~mm}$ ), to estimate the concentration $\mathrm{c}$ of the dilute solution. We took a dilution series of this solution to create an intensity standard, which we used to estimate the concentration of our expressed protein.

MinDE Self-Organization Assays. For the reconstitution of the MinDE assay on planar surfaces, we used protocols as described in earlier literature. ${ }^{22}$ For reconstitution in the chambers, a mixture of $0.5 \mu \mathrm{M}$ MinD (doped with 30\% EGFP-MinD), $1 \mu \mathrm{M}$ MinE, and 2.5 $\mathrm{mM}$ ATP (from $100 \mathrm{mM}$ ATP stock in $100 \mathrm{mM} \mathrm{MgCl}_{2}, \mathrm{pH} 7.5$ ) in buffer $\mathrm{M}$ was first prepared. The mixture was then loaded onto the flow cell and incubated for $30 \mathrm{~min}$ at room temperature $\left(23^{\circ} \mathrm{C}\right)$. The chambers were then sealed, as described previously.

\section{RESULTS AND DISCUSSION}

CYTOP Chambers Fabricated by Reactive Ion Etching and Replica Molding. Photolithography is a commonly used method to pattern materials, including CYTOP, that are 

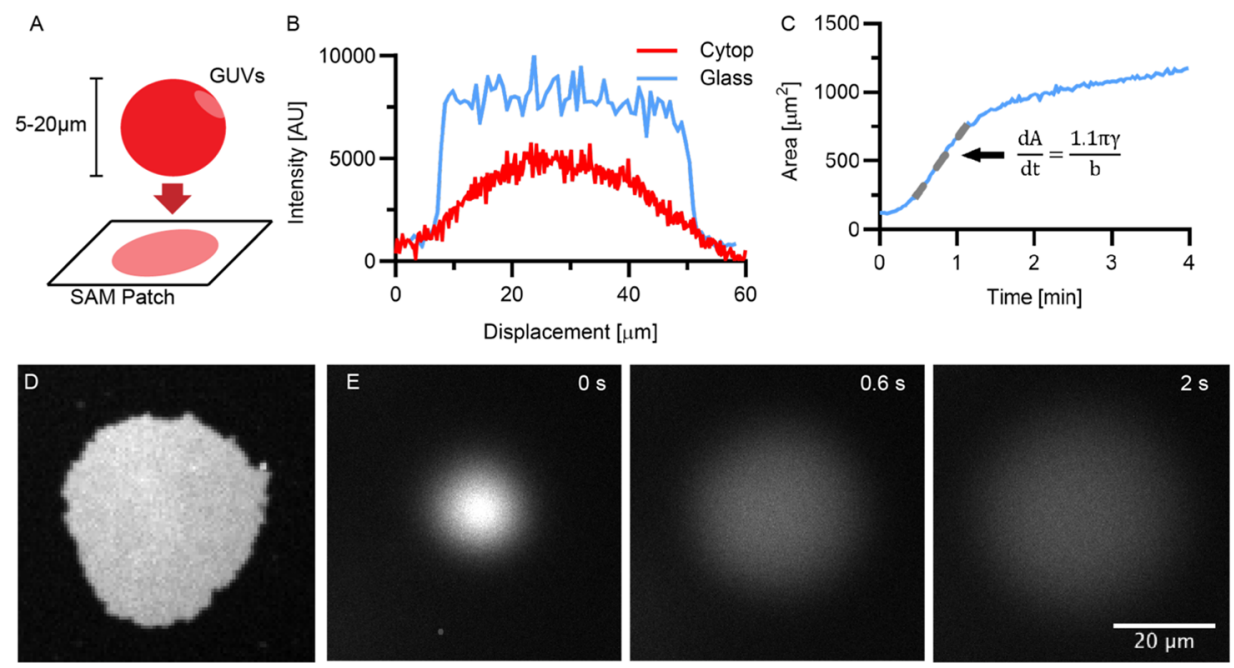

Figure 2. (A) GUVs hemifuse with CYTOP to form SAM patches. (B) Intensity profile of lipid membrane patches (taken through the diameter) formed by GUV fusion on glass (D) and on CYTOP (E). (C) Area of the membrane patch was plotted over time [from time lapse image, (E)]. From the initial gradient, we calculated the surface energy of the material $\left(21 \pm 3 \mathrm{mN} \mathrm{m}^{-1}\right)$.

themselves not photo-reactive (Figure 1A). A photoresist is first deposited on top of the material and patterned by UV light, which then becomes a mask for the subsequent chemical or plasma treatment that etches away the material in the regions that are exposed. The photoresist is then lifted off to reveal the patterned structures.

With CYTOP, the etching step is done with a high-power oxygen plasma, which requires a reactive ion etcher (RIE). Although this is a reliable and effective technique, the equipment is costly and many cleanroom facilities do not offer respective procedures. We therefore present an alternative fabrication method by replica molding (Figure 1B), which requires significantly less technical expertise and lowers equipment costs. In replica molding, ${ }^{18}$ we first require a master or a template. The master can be either purchased or fabricated by patterning a photoresist, such as SU8, by UV exposure. Then, a PDMS stamp is cast out of the master mold and pressed down on uncured CYTOP. The CYTOP is cured with the stamp in place, taking its form. Once the stamp is lifted off, a high-fidelity replica of the master remains.

The fabricated chambers by both methods were imaged on a profiler (Figure 1C, top: RIE, bottom: replica molding), and the height profile (Figure 1D) shows little difference between the two methods. In the subsequent encapsulation experiments, we used chambers produced with both methods and compared the obtained results. Replica molding is a low-cost alternative to the highly reliable photolithography method using RIE. It can, however, lead to small variations across samples. For example, there can be small damages in parts when the stamp is peeled off, and variations in the way the stamp is pressed down onto the uncured CYTOP can sometimes cause deformed structures. A rigid frame around the PDMS stamp ${ }^{28}$ would reduce deformations and increase the fidelity of the replica, and thus should be attempted in the next iteration to improve this technique.

Hemifusion of GUVs on CYTOP Confirms the Formation of SAM. To address the issue of CYTOP's biocompatibility, we attempted to coat the surface of CYTOP with lipid membrane. On hydrophobic surfaces, such as that of CYTOP, vesicles are thought to undergo the process of hemifusion, ${ }^{29}$ during which the lipids in the vesicle bilayer reorganize themselves such that the hydrophobic tails (of both the inner and outer leaflets of the vesicle) come into contact with the surface. This forms a low-energy interface between the hydrophobic substrate and the surrounding buffer, as a result of the formation of a lipid SAM.

To test the hypothesis that vesicles form a lipid monolayer upon hemifusion on the CYTOP surface, we utilized GUVs (Figure 2A). Typically with diameters 5-20 $\mu \mathrm{m}$, these membrane structures are large enough to be well resolved by optical microscopy, making them ideal test substrates for detailed analyses of the fusion process and the formed lipid patch.

We prepared a flat spin-coated CYTOP and deposited a solution containing GUVs on top of the CYTOP surface. We then observed the hemifusion process by TIRF microscopy. The SAM patch formed through GUV hemifusion resulted in a unique intensity profile on the CYTOP surface (Figure 2B) that is markedly different from that of a supported lipid bilayer (SLB) formed on glass (Figure 2D). The formed SAM patch appeared fuzzy, with a less-defined edge because of a gradual intensity fall-off that implies a sparser lipid density away from the center of the fusion site. In contrast, an SLB patch had a very defined edge with a sharp intensity fall-off, and the lipids formed a homogeneous coverage. A previous study on the hemifusion of GUVs on a hydrophobically functionalized glass surface showed that GUV-fused SAM patches have a characteristic morphology and behavior, from which we could draw close comparisons with our observations. ${ }^{29}$

We observed the hemifusion of a GUV in high time resolution ( $30 \mathrm{~ms}$ time intervals) by TIRF microscopy; we can see the vesicle coming into contact, fusing, and spreading on the CYTOP surface (Figure $3 \mathrm{E}$ ) and plotted the area increase over time (Figure 3C). We also observe the same by spinning disk confocal microscopy (Figure S1), where we can more clearly see the vesicle outline during fusion, which shrinks as lipids are reorganized and the SAM spreads over the surface. A physical model was developed by Zan et al., ${ }^{29}$ in which the instantaneous free energy gain because of the lipids spreading and the covering of the hydrophobic surface by the lipid tails is balanced by the frictional losses of the lipids flowing from the center of the hemifusion toward the edge of the covered area. 
A
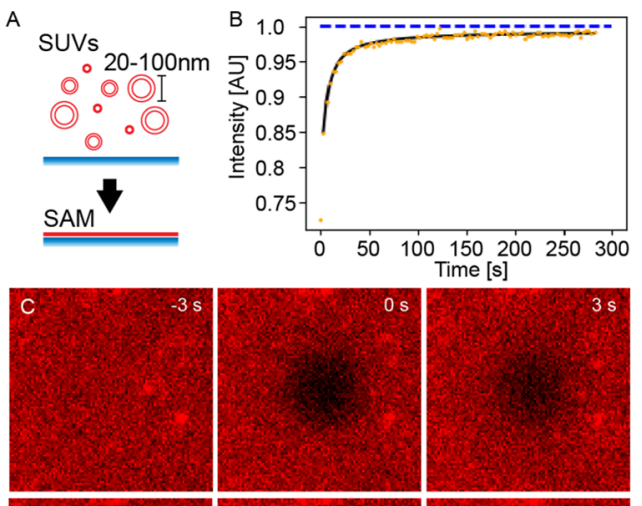

Time [s]
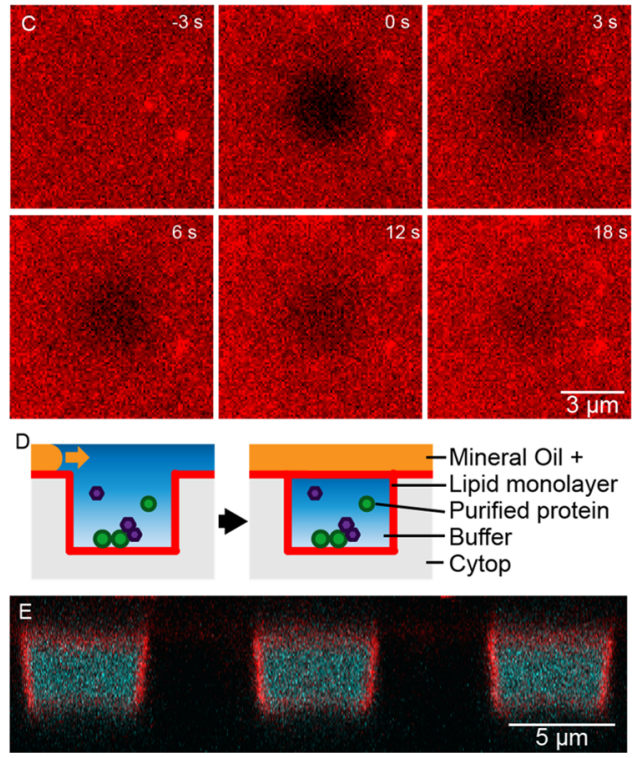

Figure 3. Lipid preparation on CYTOP surface. (A) Schematic of SAM formation. SUVs were incubated on the CYTOP surface, where they fuse to form a homogeneous SAM. (B,C) Subsequent FRAP analysis shows fluorescence recovery. (D) After coating the surface with SAM, the chambers can be sealed by flushing in mineral oil containing lipids through a flow cell. (E) $Z$-stacks of the sealed chambers were taken with a confocal microscope. The lipids are labeled red (DOPE-Atto655) and soluble Alexa 488 dye in the encapsulated volume (cyan).

For vesicles with radii $R \approx 10 \mu \mathrm{m}$, the initial rate of area expansion can be approximated by a linear equation ${ }^{29}$

$$
\frac{\mathrm{d} A}{\mathrm{~d} t} \approx \frac{1.1 \pi \gamma}{b}
$$

where $\gamma$ is the surface energy, $b$ is the friction coefficient, and $A$ is the area over which the lipid layer spreads. Using the value $b$ $\approx 10^{8} \mathrm{~N} \mathrm{~s} \mathrm{~m}^{-3}$ (based on Zan et al. ${ }^{29}$ ) and taking the initial linear gradient from the first $\sim 500 \mathrm{~ms}$ of area expansion after fusion occurs, we calculated the surface energy of the material $\left(21 \pm 3 \mathrm{mN} \mathrm{m}^{-1}\right)$, which is in good agreement with previous measurements (19 $\left.\mathrm{mN} \mathrm{m}^{-1}\right)$, published by Asahi-Glass. ${ }^{11}$ Taken together, our results support that the CYTOPsupported lipid membrane is indeed a monolayer.

Self-Assembled Lipid Monolayers Formed on CYTOP through SUV Fusion. Having obtained strong evidence for the formation of SAMs on CYTOP, we attempted to form a uniform coating of lipids on the fabricated chamber surface. For this purpose, we deposited a solution containing a high concentration of SUVs on top of the CYTOP surface (Figure 3A). We prepared the SUVs with two lipid compositions: one containing DOPC and another with a DOPC/DOPG mixture $(7: 3)$. The latter has a net negative charge, which is crucial for the function of many membrane-interacting proteins, such as E. coli MinDE.
Both RIE-prepared and replica-molded structures were successfully coated with a homogeneous SAM (Figure S2), whose membrane mobility was assessed by FRAP (Figure $3 \mathrm{~B}, \mathrm{C})$. In all cases, the obtained lipid diffusion coefficients were between 1.0 and $1.5 \mu \mathrm{m}^{2} \mathrm{~s}^{-1}$ (Table 1), which are

Table 1. Calculated Diffusion Coefficients from FRAP Analysis $^{a}$

\begin{tabular}{lccccc} 
& \multicolumn{2}{c}{$\begin{array}{c}\text { diffusion coefficient } \\
\left(\mu \mathrm{m}^{2} \mathrm{~s}^{-1}\right)\end{array}$} & & \multicolumn{2}{c}{ mobile fraction $(\%)$} \\
\cline { 2 - 3 } \cline { 5 - 6 } & RIE & replica & & RIE & replica \\
DOPC & $1.3 \pm 0.3$ & $1.5 \pm 0.7$ & & $98 \pm 2$ & $97 \pm 3$ \\
DOPC/DOPG & $1.0 \pm 0.2$ & $1.3 \pm 0.5$ & & $97 \pm 3$ & $97 \pm 2$
\end{tabular}

${ }^{a}$ Error represents standard deviation of measurement values from $n=$ 15 independent experiments.

comparable to those of other SAM systems $\left(0.2-2 \mu \mathrm{m}^{2} \mathrm{~s}^{-1}\right)$, $\backslash^{30,31}$ and still in the range of those measured for SLBs $(1-6$ $\left.\mu \mathrm{m}^{2} \mathrm{~s}^{-1}\right) .32,33$ More importantly, the immobile fraction was negligible, indicating the lack of major lipid aggregates and other surface artifacts.

Having fabricated the chambers and coated their surfaces with lipid membranes, we encapsulated biochemical reactions. The loading of the chambers was done in a flow cell, as shown in the schematic (Figure 3D). The confocal cross-sectional image (Figures 3E and S3) shows Alexa 488 dye successfully encapsulated in these lipid-coated chambers.

SAM on CYTOP Improves Biocompatibility. The native CYTOP surface is incompatible with many biological systems. Here, we show that SAM coverage can provide adequate passivation, which increases the biocompatibility of our CYTOP system. We first used water-soluble eGFP as a model protein. Despite its apparent hydrophilicity, eGFP adheres to and denatures upon contact with the CYTOP surface (Figure S4). Hence, we attempted to show that the SAM passivation is sufficient to shield the protein from CYTOP's hydrophobic surface.

A solution containing $50 \mathrm{nM}$ eGFP was incubated on top of CYTOP (Figure S4A). Without the SAM, the fluorescence intensity on the surface was almost 16-fold higher than in the solution (Figure S4C,D), and we also saw aggregates forming on the surface. We verified that there was almost no recovery after photobleaching, suggesting that the eGFP attached permanently to the surface (Figure S4E,F). We then formed the SAM (DOPC) prior to depositing the eGFP solution. In this case, the fluorescence intensity on the surface could be dramatically reduced (Figure S4B-D). We still found a slight increase in the fluorescence on the membrane, which could point to a weak interaction between eGFP and the lipid. In contrast to the non-passivated CYTOP surface, eGFP was not permanently bound, as seen from the complete fluorescence recovery after bleaching on the surface.

To further verify the enhanced biocompatibility, we attempted cell-free protein synthesis (CFPS) inside the SAM-covered CYTOP chambers. The encapsulation of cellfree extracts has recently caught much attention, ${ }^{34}$ because proteins can be expressed in defined volumes without the need of any additional protein purification steps. ${ }^{35}$ The cell-free extract is an extremely complex mixture of proteins and cofactors and can therefore be compromised by the incompatibility of its constituents with their surrounding material. Moreover, encapsulating volumes inside small chambers, 
whose surface area-to-volume ratio is high, emphasizes this problem; adsorption onto the larger surface to available volume can lead to a more significant depletion of components away from the reaction volume. Here, we show that GFP can be readily expressed within SAM-covered chambers, as the increased biocompatibility because of the SAM allows protein synthesis to occur efficiently.

The chambers were fabricated by the two alternative methods outlined before (photolithography and replica molding). The SAM was formed on the surface, and the chambers were subsequently loaded with the cell-free extract. After sealing the chambers, we incubated them at $37{ }^{\circ} \mathrm{C}$ over 5 $\mathrm{h}$, monitoring the GFP expression levels by measuring the fluorescence intensity in the fabricated wells every $30 \mathrm{~min}$ (Figure 4).

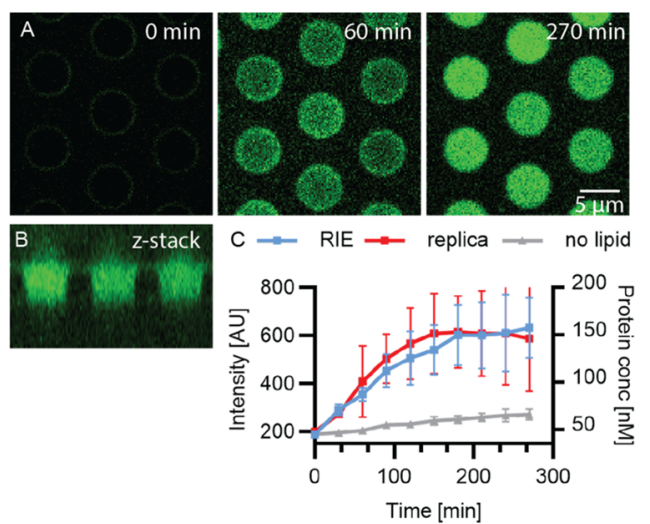

Figure 4. GFP expression by CFPS in sealed CYTOP chambers. (A) Time lapse images of the chambers, with time intervals of $30 \mathrm{~min}$. (B) $Z$-stack image of the sealed chambers. (C) GFP expression levels against incubation time at $37{ }^{\circ} \mathrm{C}$. Error bars represent standard deviation of measurement values from $n=5$ independent experiments.

In our chambers, GFP was expressed almost linearly for the first $2 \mathrm{~h}$ of incubation, after which the expression slowed down. Without lipid passivation, GFP levels stayed low even after $5 \mathrm{~h}$ of incubation. This is a strong indication that the SAM's passivation is effective at preventing essential cell-free components from adsorbing to the CYTOP surface.

In this experimental set-up, each glass slide contains around 100000 chambers of picoliter-sized reaction volumes. In our flow cell, the required sample volume used for loading was as low as $40 \mu \mathrm{L}$. The high-throughput, yet highly efficient, nature of these microfabricated systems can be further improved by connecting it to a microfluidic set-up, and this could also allow more complex exchange of solutions, to enable, for example, the generation of concentration gradients over the slide. ${ }^{36}$

The expression of proteins using cell-free extracts in such high-throughput systems can be a powerful technique. Directed evolution of enzymes, where DNA from a DNA library could be introduced to each chamber and directly expressed in the cell-free extract, is an example. We also speculate that this technique can be combined with ALBiCstyle chambers, developed by Noji et al., ${ }^{37}$ where, instead of having a mineral oil seal, the chambers have a free-standing bilayer interface sealing the top of the chamber.

SAM on CYTOP as a Platform for the Study of Membrane-Interacting Protein Systems: E. coli MinDE. We investigated whether the lipid interface formed on CYTOP can be used as a platform to study membrane-interacting proteins. In particular, we investigated the E. coli MinDE system, which is known to form dynamic patterns when reconstituted on supported lipid membranes. ${ }^{38}$ The reconstitution of the Min system on planar surfaces, as well as encapsulation in aspherical compartments, has been well studied, ${ }^{6,10,39,40}$ and therefore, it is an ideal model protein system to test our new platform. Importantly, either the micropatterning methods employed in previous studies have not succeeded in fully sealing the reaction volume ${ }^{10}$ or the structural material they have used can have unfavorable chemical or optical properties, ${ }^{7}$ particularly in the case of PDMS.

The E. coli MinDE system involves two proteins, MinD and MinE: MinD forms a dimer in the presence of ATP, upon which it attaches cooperatively to the membrane. At higher local concentrations on the membrane, it recruits MinE. MinD's ATPase activity gets activated when MinE is bound to it, which in turn triggers its detachment from the membrane. ${ }^{41}$ The time delay between the accumulation of $\mathrm{MinD}$ on the membrane and MinE-triggered detachment, or in other words, the delayed negative feedback, results in traveling waves of Min proteins on a planar surface, ${ }^{39,40}$ and pole-to-pole oscillations when confined in a cylindrical geometry, ${ }^{10}$ which has been shown to position the cell division ring in E. coli. ${ }^{42}$

First, we prepared a planar CYTOP surface by spin-coating and coated the surface by SUV hemifusion (lipid composition DOPC/DOPG in 7:3 ratio). We then incubated purified MinDE proteins and observed their dynamics. Dynamic spiral patterns were observed on the surface (Figures 5A and S5), in good qualitative agreement with observations made in previous studies on supported bilayers. ${ }^{39}$ The measured period (1-2 min) and wavelength $(\sim 30 \mu \mathrm{m})$ compare well with previous studies on bilayers (period $0.6-2 \mathrm{~min}$, wavelength $50-110$ $\mu \mathrm{m})^{39,40,43}$ as well as on monolayer surfaces at the air-water interface (period 0.5-1 min, wavelength $30-60 \mu \mathrm{m}$ ). ${ }^{44}$ The Min wavelength is known to vary according to the substrate and its preparation, ${ }^{43}$ and a different wavelength on CYTOP can therefore be expected.

As a control, we checked that MinD is indeed adhering to and denaturing on CYTOP without the SAM (Figure 5B). Without MinE and in the presence of ATP, MinD still has a basal on/off rate from the membrane that is not catalyzed by MinE binding. Without the SAM, FRAP analysis on this system shows only partial recovery, which suggests that most of the MinD is permanently bound to the CYTOP surface. With the SAM, MinD fluorescence almost fully recovers, which indicates a healthy cycling of $\mathrm{MinD}$ from the membrane into the bulk volume.

We fabricated chambers as bacteria mimicries - in elongated chambers with aspect ratios (width/length) ranging from 1:1 to 1:5. Both photolithography (Figure 5) and replica molding (Figure S6) methods were tested, and we confined the MinDE proteins within these chambers and observed their dynamics.

After encapsulation, MinDE performed pole-to-pole oscillations in the longer chambers, in good agreement with previous in vitro studies. ${ }^{6,10}$ These standing waves determine midcell in E. coli: ${ }^{45}$ the time-averaged concentration of MinD is depleted in the middle, and this is where the FtsZ ring can form and act as a scaffold for subsequent assembly of the cell division machinery. In small aspect ratio chambers, we also observed volume oscillations or "blinking" of Min proteins, where the Min proteins are periodically either attached to or 

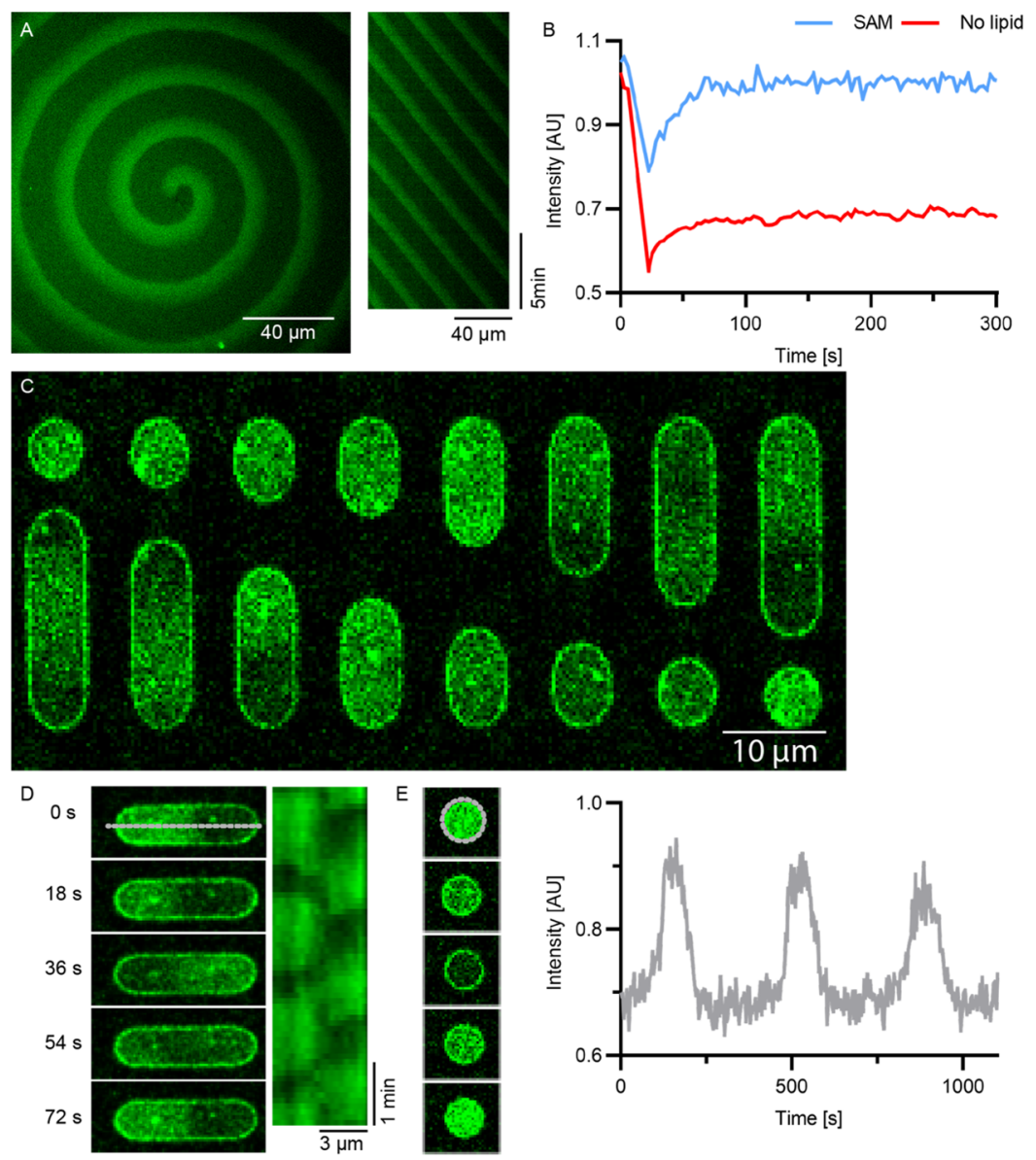

Figure 5. MinDE system dynamics reconstituted on CYTOP-SAM. (A) Min proteins form spiral waves on a planar surface. (B) As a control, MinD activity on the surface was checked by FRAP with and without the SAM. (C) Min proteins can be encapsulated in rod-shaped chambers, with aspect ratios ranging from 1:1-5. (D) Time lapse images of pole-to-pole oscillations in rod-shaped chambers and (E) "blinking" dynamics in symmetrical compartments.

dissociated from the membrane. This behavior was also seen previously in spherical vesicles and in systems with high surface/volume ratios. ${ }^{44,46}$

The successful encapsulation of the MinDE system conveys two important messages with regard to other potential applications of the CYTOP structures described here. The first is that complex protein systems, whose function is dependent on geometry, can be readily studied in these confined and asymmetrical chambers when they are passivated by a lipid membrane. The second is that a negatively charged lipid membrane can be formed on the surface, providing an active and catalytic interface that is essential for the function of many proteins. Indeed, most prokaryotic cell membranes are negatively charged, and therefore, many bacterial protein systems are adapted to work on this type of membrane. A more comprehensive investigation of lipid species, such as $\mathrm{PIP}_{2}$ or DOPS, could further extend the range of protein systems (e.g., eukaryotic) that CYTOP-SAM chambers can be utilized for.

\section{CONCLUSIONS}

In the search for new materials for the fabrication of biomimetic and biocompatible compartments to encapsulate complex protein systems, we turned our attention to CYTOP, an amorphous fluorinated polymer that has good chemical resistance and ideal optical properties for imaging with standard fluorescence microscopy methods. We demonstrated that CYTOP chambers can be fabricated by two methods: one, by photolithography using RIE, and second, by replica molding. In order to increase the range of compatible biosystems, we deposited an SAM on the CYTOP surface by SUV fusion. By coating it with lipid monolayers, the surface can be efficiently passivated against adhesion and subsequent denaturation of proteins. Importantly, the structures can be fully sealed by a lipid monolayer, enabling the design of closed membrane compartments of defined 3D geometry. Furthermore, we demonstrate that the CYTOP-SAM is an improved model membrane platform to study membrane-interacting proteins, in particular the dynamic MinDE system.

\section{ASSOCIATED CONTENT}

\section{S Supporting Information}

The Supporting Information is available free of charge on the ACS Publications website at DOI: 10.1021/acsami.9b05073.

GUV hemifusion time lapse, homogeneous SAM on CYTOP, Z-stack images of sealed chambers, eGFP adhesion on CYTOP, MinDE oscillation on planar surface, and MinDE oscillations on replica molded chambers (PDF)

Movie of GUV hemifusion on CYTOP (AVI) 


\section{AUTHOR INFORMATION}

\section{Corresponding Author}

*E-mail: schwille@biochem.mpg.de.

\section{ORCID $\odot$}

Hiromune Eto: 0000-0001-8737-2939

Henri G. Franquelim: 0000-0001-6229-4276

Philipp Glock: 0000-0002-0238-2634

Alena Khmelinskaia: 0000-0003-1584-9800

Hiroyuki Noji: 0000-0002-8842-6836

Petra Schwille: 0000-0002-6106-4847

\section{Author Contributions}

H.E. contributed to the project design, conducted experiments, analyzed data, and wrote the article. N.S. conducted experiments on chamber fabrication. H.G.F. conducted experiments on GUV hemifusion and FRAP analysis and wrote the article. L.K. conducted experiments on CFPS and wrote the article. M.H. contributed to the project design. P.G. and A.K. conducted experiments on monolayer preparation and wrote the article. P.G. also conducted experiments on dynamic protein reconstitution assays. H.N. and P.S. contributed to the project design and wrote the article.

\section{Notes}

The authors declare no competing financial interest.

\section{ACKNOWLEDGMENTS}

We thank Allen Liu for discussion and helpful comments on the article, the Biochemistry Core Facility of the Max Planck Institute of Biochemistry for assistance with protein purification, as well as the support from Center for Nanosciences, Munich. P.G. acknowledges support from the DFGfunded GRK 2062-Molecular Principles of Synthetic Biology as well as support from International Max Planck Research School for Molecular Life Sciences. A.K. acknowledges the support of the Graduate School of Quantitative Biosciences Munich. P.S. acknowledges the financial support from the Max Planck Society (MPG) and from the DFG through the project A09 of the SFB1032 "Nanoagents for the spatiotemporal control of molecular and cellular reactions". H.G.F. and P.S. acknowledge the financial support from project B10 of the SFB863 "Forces in Biomolecular Systems". L.K., M.H., and P.S. have received funding from the MaxSynBio Consortium, which is jointly funded by the Federal Ministry of Education and Research of Germany (BMBF) and MPG. N.S. acknowledges the financial support from Grants-in-Aid for Scientific Research (KAKENHI) 17H05717 from the Japan Society for the Promotion of Science. H.N. acknowledges the financial support from the Japan Science and Technology Agency for ImPACT Program of Council for Science, Technology, and Innovation (Cabinet Office, Government of Japan) to K. V. T. (grant number: 15808948) as well as from JST CREST grant number JPMJCR18S6.

\section{REFERENCES}

(1) Sezgin, E.; Levental, I.; Mayor, S.; Eggeling, C. The Mystery of Membrane Organization: Composition, Regulation and Roles of Lipid Rafts. Nat. Rev. Mol. Cell Biol. 2017, 18, 361-374.

(2) Dezi, M.; Di Cicco, A.; Bassereau, P.; Levy, D. Detergentmediated incorporation of transmembrane proteins in giant unilamellar vesicles with controlled physiological contents. Proc. Natl. Acad. Sci. U.S.A. 2013, 110, 7276-7281.
(3) Yamada, A.; Lee, S.; Bassereau, P.; Baroud, C. N. Trapping and Release of Giant Unilamellar Vesicles in Microfluidic Wells. Soft Matter 2014, 10, 5878-5885.

(4) Korlach, J.; Reichle, C.; Müller, T.; Schnelle, T.; Webb, W. W. Trapping, Deformation, and Rotation of Giant Unilamellar Vesicles in Octode Dielectrophoretic Field Cages. Biophys. J. 2005, 89, 554-562.

(5) Yeaman, C.; Grindstaff, K. K.; Nelson, W. J. New Perspectives on Mechanisms Involved in Generating Epithelial Cell Polarity. Physiol. Rev. 1999, 79, 73-98.

(6) Zieske, K.; Schwille, P. Reconstitution of Self-Organizing Protein Gradients as Spatial Cues in Cell-Free Systems. eLife 2014, 3, No. e03949.

(7) Caspi, Y.; Dekker, C. Mapping out Min Protein Patterns in Fully Confined Fluidic Chambers. eLife 2016, 5, No. e19271.

(8) Bao, M.; Xie, J.; Piruska, A.; Huck, W. T. S. 3D Microniches Reveal the Importance of Cell Size and Shape. Nat. Commun. 2017, 8, $1-12$.

(9) Weibel, D. B.; Diluzio, W. R.; Whitesides, G. M. Microfabrication Meets Microbiology. Nat. Rev. Microbiol. 2007, 5, 209218.

(10) Zieske, K.; Schwille, P. Reconstitution of Pole-to-Pole Oscillations of Min Proteins in Microengineered Polydimethylsiloxane Compartments. Angew. Chem., Int. Ed. 2013, 52, 459-462.

(11) Asahi Glass Co. Ltd. J. Cytop Technical Report.

(12) Nakahara, A.; Shirasaki, Y.; Kawai, K.; Ohara, O.; Mizuno, J.; Shoji, S. Fabrication of High-Aspect-Ratio Amorphous Perfluorinated Polymer Structure for Total Internal Reflection Fluorescence Microscopy. Microelectron. Eng. 2011, 88, 1817-1820.

(13) Lee, J. N.; Park, C.; Whitesides, G. M. Solvent Compatibility of Poly(dimethylsiloxane)-Based Microfluidic Devices. Anal. Chem. 2003, 75, 6544-6554.

(14) Kimura, H.; Yamamoto, T.; Sakai, H.; Sakai, Y.; Fujii, T. An Integrated Microfluidic System for Long-Term Perfusion Culture and on-Line Monitoring of Intestinal Tissue Models. Lab Chip 2008, 8, $741-746$.

(15) Heymann, M.; Opthalage, A.; Wierman, J. L.; Akella, S.; Szebenyi, D. M. E.; Gruner, S. M.; Fraden, S. Room-Temperature Serial Crystallography Using a Kinetically Optimized Microfluidic Device for Protein Crystallization and on-Chip X-Ray Diffraction. IUCrJ 2014, 1, 349-360.

(16) Watanabe, R.; Soga, N.; Fujita, D.; Tabata, K. V.; Yamauchi, L.; Hyeon Kim, S.; Asanuma, D.; Kamiya, M.; Urano, Y.; Suga, H.; Noji, H. Arrayed Lipid Bilayer Chambers Allow Single-Molecule Analysis of Membrane Transporter Activity. Nat. Commun. 2014, 5, 4519.

(17) Lagny, T. J.; Bassereau, P. Bioinspired Membrane-Based Systems for a Physical Approach of Cell Organization and Dynamics: Usefulness and Limitations. Interface Focus 2015, 5, 20150038.

(18) Gicquel, Y.; Schubert, R.; Kapis, S.; Bourenkov, G.; Schneider, T.; Perbandt, M.; Betzel, C.; Chapman, H. N.; Heymann, M. Microfluidic Chips for In Situ Crystal X-Ray Diffraction and In Situ Dynamic Light Scattering for Serial Crystallography. J. Visualized Exp. 2018, 134, No. e57133.

(19) Watanabe, R.; Soga, N.; Ohdate, S.; Noji, H. Single-Molecule Analysis of Membrane Transporter Activity by Means of a Microsystem. In Bacterial Multidrug Exporters: Methods and Protocols; Yamaguchi, A., Nishino, K., Eds.; Springer New York: New York, NY, 2018; pp 321-330.

(20) Angelova, M. I.; Dimitrov, D. S. Liposome Electroformation. Faraday Discuss. Chem. Soc. 1986, 81, 303-311.

(21) García-Sáez, A. J.; Carrer, D. C.; Schwille, P. Fluorescence Correlation Spectroscopy for the Study of Membrane Dynamics and Organization in Giant Unilamellar Vesicles. In Liposomes: Methods and Protocols, Volume 2: Biological Membrane Models; Weissig, V., Ed.; Humana Press: Totowa, NJ, 2010; pp 493-508.

(22) Ramm, B.; Glock, P.; Schwille, P. In Vitro Reconstitution of Self-Organizing Protein Patterns on Supported Lipid Bilayers. J. Visualized Exp. 2018, 137, No. e58139.

(23) Schindelin, J.; Arganda-Carreras, I.; Frise, E.; Kaynig, V.; Longair, M.; Pietzsch, T.; Preibisch, S.; Rueden, C.; Saalfeld, S.; 
Schmid, B.; Tinevez, J. Y.; White, D. J.; Hartenstein, V.; Eliceiri, K.; Tomancak, P.; Cardona, A. Fiji: An Open-Source Platform for Biological-Image Analysis. Nat. Methods 2012, 9, 676-682.

(24) Soumpasis, D. Theoretical Analysis of Fluorescence Photobleaching Recovery Experiments. Biophys. J. 1983, 41, 95-97.

(25) Kitagawa, T.; Yabuki, T.; Yoshida, Y.; Tsutsui, M.; Ito, Y.; Shibata, T.; Yokoyama, S. Cell-Free Production and Stable-Isotope Labeling of Milligram Quantities of Proteins. FEBS Lett. 1999, 442, $15-19$.

(26) Kai, L.; Roos, C.; Haberstock, S.; Proverbio, D.; Ma, Y.; Junge, F.; Karbyshev, M.; Dötsch, V.; Bernhard, F. Systems for the Cell-Free Synthesis of Proteins. In Chemical Genomics and Proteomics: Reviews and Protocols; Zanders, E. D., Ed.; Humana Press: Totowa, NJ, 2012; pp 201-225.

(27) Kai, L.; Dötsch, V.; Kaldenhoff, R.; Bernhard, F. Artificial Environments for the Co-Translational Stabilization of Cell-Free Expressed Proteins. PLoS One 2013, 8, No. e56637.

(28) Aghvami, S. A.; Opathalage, A.; Zhang, Z. K.; Ludwig, M.; Heymann, M.; Norton, M.; Wilkins, N.; Fraden, S. Rapid Prototyping of Cyclic Olefin Copolymer (COC) Microfluidic Devices. Sens. Actuators, B 2017, 247, 940-949.

(29) Zan, G. H.; Tan, C.; Deserno, M.; Lanni, F.; Lösche, M. Hemifusion of Giant Unilamellar Vesicles with Planar Hydrophobic Surfaces: A Fluorescence Microscopy Study. Soft Matter 2012, 8, 10877-10886.

(30) Yang, Z.; Yu, H. Biomembrane Mimetic Surfaces by Phospholipid Self-Assembled Monolayers on Silica Substrates. Langmuir 1999, 15, 1731-1737.

(31) Yeung, S. Y.; Ederth, T.; Pan, G.; Cicenaite, J.; Cárdenas, M.; Arnebrant, T.; Sellergren, B. Reversible Self-Assembled Monolayers (rSAMs) as Robust and Fluidic Lipid Bilayer Mimics. Langmuir 2018, 34, 4107-4115.

(32) Chiantia, S.; Ries, J.; Kahya, N.; Schwille, P. Combined AFM and Two-Focus SFCS Study of Raft-Exhibiting Model Membranes. ChemPhysChem 2006, 7, 2409-2418.

(33) Tamm, L. K.; McConnell, H. M. Supported Phospholipid Bilayers. Biophys. J. 1985, 47, 105-113.

(34) Jia, H.; Heymann, M.; Bernhard, F.; Schwille, P.; Kai, L. CellFree Protein Synthesis in Micro Compartments: Building a Minimal Cell from Biobricks. New Biotechnol. 2017, 39, 199-205.

(35) Majumder, S.; Garamella, J.; Wang, Y.-L.; Denies, M.; Noireaux, V.; Liu, A. P. Cell-Sized Mechanosensitive and Biosensing Compartment Programmed with DNA. Chem. Commun. 2017, 53, $7349-7352$.

(36) Jeon, N. L.; Dertinger, S. K. W.; Chiu, D. T.; Choi, I. S.; Stroock, A. D.; Whitesides, G. M. Generation of Solution and Surface Gradients Using Microfluidic Systems. Langmuir 2000, 16, 83118316.

(37) Watanabe, R.; Soga, N.; Hara, M.; Noji, H. Arrayed Water-inOil Droplet Bilayers for Membrane Transport Analysis. Lab Chip 2016, 16, 3043-3048.

(38) Loose, M.; Fischer-Friedrich, E.; Ries, J.; Kruse, K.; Schwille, P. Spatial Regulators for Bacterial Cell Division Self-Organize into Surface Waves in Vitro. Science 2008, 320, 789-792.

(39) Loose, M.; Fischer-Friedrich, E.; Ries, J.; Kruse, K.; Schwille, P. Spatial Regulators for Bacterial Cell Division Self-Organize into Surface Waves in Vitro. Science 2008, 320, 789-792.

(40) Loose, M.; Fischer-Friedrich, E.; Herold, C.; Kruse, K.; Schwille, P. Min Protein Patterns Emerge from Rapid Rebinding and Membrane Interaction of MinE. Nat. Struct. Mol. Biol. 2011, 18, 577583.

(41) Kretschmer, S.; Schwille, P. Pattern Formation on Membranes and Its Role in Bacterial Cell Division. Curr. Opin. Cell Biol. 2016, 38, 52-59.

(42) Raskin, D. M.; de Boer, P. A. J. Rapid Pole-to-Pole Oscillation of a Protein Required for Directing Division to the Middle of Escherichia Coli. Proc. Natl. Acad. Sci. U.S.A. 1999, 96, 4971-4976.
(43) Martos, A.; Petrasek, Z.; Schwille, P. Propagation of MinCDE Waves on Free- Standing Membranes. Environ. Microbiol. 2013, 15, 3319-3326.

(44) Zieske, K.; Chwastek, G.; Schwille, P. Protein Patterns and Oscillations on Lipid Monolayers and in Microdroplets. Angew. Chem., Int. Ed. 2016, 55, 13455-13459.

(45) Raskin, D. M.; de Boer, P. A. J. Rapid Pole-to-Pole Oscillation of a Protein Required for Directing Division to the Middle of Escherichia Coli. Proc. Natl. Acad. Sci. U.S.A. 1999, 96, 4971-4976.

(46) Litschel, T.; Ramm, B.; Maas, R.; Heymann, M.; Schwille, P. Beating Vesicles: Encapsulated Protein Oscillations Cause Dynamic Membrane Deformations. Angew. Chem., Int. Ed. 2018, 57, 1628616290. 